\title{
The robust optimisation of metal forming processes
}

\author{
M.H.A. Bonte, A.H. van den Boogaard and R. van Ravenswaaij \\ University of Twente, P.O. Box 217, 7500 AE Enschede, The Netherlands
}

\begin{abstract}
Robustness, reliability, optimisation and Finite Element simulations are of major importance to improve product quality and reduce costs in the metal forming industry. In this paper, we review several possibilities for combining these techniques and propose a robust optimisation strategy for metal forming processes. The importance of including robustness during optimisation is demonstrated by applying the robust optimisation strategy to an analytical test function: for constrained cases, deterministic optimisation will yield a scrap rate of about $50 \%$ whereas the robust counterpart reduced this to the required $3 \sigma$ reliability level.
\end{abstract}

Keywords: optimisation, metal forming, FEM, robustness, reliability

\section{INTRODUCTION}

Product improvement and cost saving have always been important goals in the metal forming industry. One way of achieving these two goals is optimising towards robust metal forming processes. A robust metal forming process will yield metal products at a more constant quality level. Hence, it will (i) improve the product' quality; and (ii) save costs because the number of non-feasible products (scrap) is decreased. Generally, optimisation strategies only include deterministic control variables. To assess the robustness of a metal forming process, the noise variables (e.g. material variation) need to be taken into account during optimisation.

In [1], we presented three ways to optimise towards robust metal forming processes using time consuming Finite Element simulations of these processes: deterministic optimisation, robust optimisation and reliability based optimisation. An example of deterministic optimisation to yield a robust deep drawing process has been included in the same paper. In this paper, we continue on robust optimisation techniques. We review several possibilities for optimising towards robust metal forming processes and present a robust optimisation strategy that includes both design and noise variables into optimisation. The robust optimisation strategy is demonstrated and compared to deterministic optimisation by application to an analytical test function.

\section{POSSIBILITIES FOR ROBUST OPTIMISATION}

From the three possibilities published in [1], we propose to continue with robust optimisation techniques. This is based on the following considerations:

- Deterministic optimisation does not take into account process robustness and reliability during optimisation; 
- Reliability based optimisation algorithms are generally very time consuming;

- Robust optimisation takes into account process robustness and even reliability if a response distribution is assumed (e.g. a normal distribution);

- Robust optimisation is relatively efficient.

Two ways for robust optimisation are the Taguchi and Dual Response Surface Methods [2]. Taguchi methods are based on crossed orthogonal array Design Of Experiments (DOE) plans. After having run the physical or in our case numerical experiments, response measurements can be analysed based on ANalysis Of VAriance (ANOVA) and Signal-to-Noise ratios ( $\mathrm{S} / \mathrm{N}$-ratios). Taguchi methods possess several severe disadvantages [3, 4]: (i) Use of the $\mathrm{S} / \mathrm{N}$-ratio implies the mean and variance of a response distribution are confounded; (ii) crossed orthogonal arrays lack flexibility and efficiency; (iii) Taguchi methods do not allow for sequential experimentation/optimisation.

Alternatively one can employ Dual Response Surface Methods (DRSM): one Response Surface Model (RSM) is fitted for the mean and one for the variance of a response [2]. The most straight-forward way of robust optimisation using DRSM is Direct Variance Modelling: for each of the DOE points in the control variable space, one can perform an orthogonal array in the noise variable space to assess the probability distribution of the response for those control variable settings. Basically, this resembles Taguchi methods, but overcomes the three disadvantages mentioned above. Direct Variance Modelling is, however, very time consuming since noise variable assessment requires performing several FEM calculations for each control variable setting.

A much more efficient way of robust optimisation using DRSM is fitting one single RSM metamodel in both the control and noise design variable space, e.g. the following RSM metamodel which is quadratic in the design variable space and linear + interaction in the noise variable space:

$$
\hat{y}(\mathbf{x}, \mathbf{z})=\beta_{0}+\mathbf{x}^{\mathrm{T}} \beta+\mathbf{x}^{\mathrm{T}} \mathbf{B} \mathbf{x}+\mathbf{z}^{\mathrm{T}} \gamma+\mathbf{x}^{\mathrm{T}} \Delta \mathbf{z}+\varepsilon
$$

where $\hat{y}$ is a single metamodel of a response dependent on the control variables $\mathbf{x}$ and noise variables $\mathbf{z} . \beta_{0}, \beta, \mathbf{B}, \gamma$ and $\Delta$ denote the fitted regression coefficients and $\varepsilon$ is the random error term. From Equation 1, one can analytically determine two RSM metamodels for mean and variance [2]:

$$
\begin{array}{r}
\mu_{y}=E[\hat{y}(\mathbf{x}, \mathbf{z})]=\beta_{0}+\mathbf{x}^{\mathrm{T}} \beta+\mathbf{x}^{\mathrm{T}} \mathbf{B} \mathbf{x} \\
\sigma_{y}^{2}=\operatorname{var}[\hat{y}(\mathbf{x}, \mathbf{z})]=\sigma_{z}^{2}\left(\gamma^{\mathrm{T}}+\mathbf{x}^{\mathrm{T}} \Delta\right)\left(\gamma+\Delta^{\mathrm{T}} \mathbf{x}\right)+\sigma^{2}
\end{array}
$$

with $\mu_{y}$ and $\sigma_{y}^{2}$ the metamodels for mean and variance of the response. Overcoming the disadvantages of the Taguchi method and being more efficient than Direct Variance Modelling, we propose a robust optimisation strategy based on fitting single response surfaces in the combined control-noise variable space. 


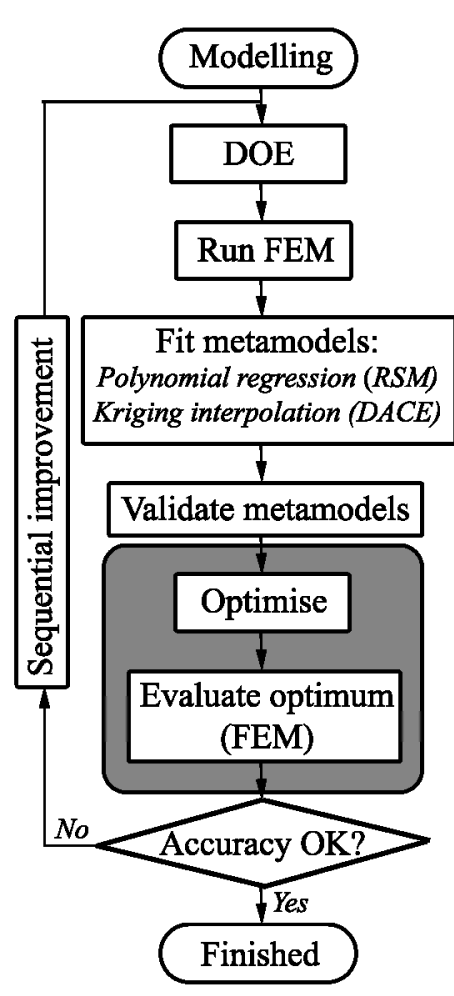

(a)

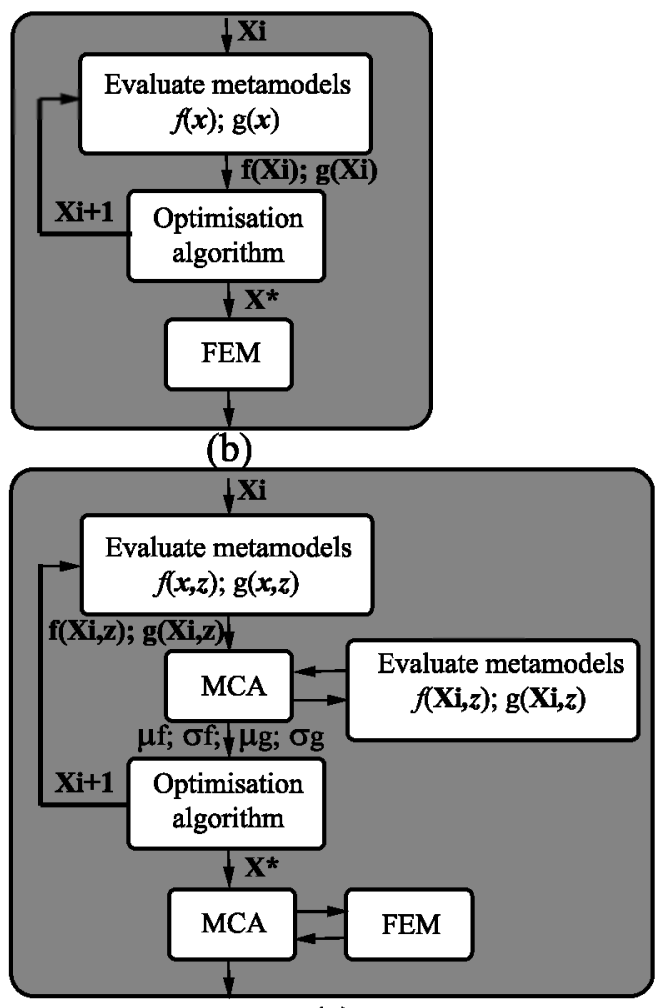

(c)

FIGURE 1. (a) Flow chart of the optimisation strategy; (b) Deterministic; (c) Robust

\section{A ROBUST OPTIMISATION STRATEGY FOR METAL FORMING PROCESSES}

The proposed robust optimisation strategy is an extension of a deterministic optimisation strategy for metal forming processes presented in amongst others [5]. A flowchart of the optimisation strategy is presented in Figure 1(a). The robust optimisation strategy differs from the deterministic strategy in the modelling, optimisation and evaluation parts.

Concerning the modelling, noise variables are included in addition to deterministic control variables. For the noise variables, a normal distribution is assumed. For each response (objective function or constraint), one now obtains a response distribution $\left(\mu_{y}\right.$ and $\sigma_{y}$ ) instead of a response value $y$. As objective function $f$ one can optimise $\mu_{f}$, $\sigma_{f}$ or a weighted sum $\mu_{f} \pm w \sigma_{f}$. If $\mu_{f}$ or $\sigma_{f}$ are optimised, it is advised to include the weighted sum as a constraint: this takes into account process reliability in the optimisation problem. Also other constraints $g$ are taken into account as a weighted $\operatorname{sum} \mu_{g} \pm w \sigma_{g}$. 


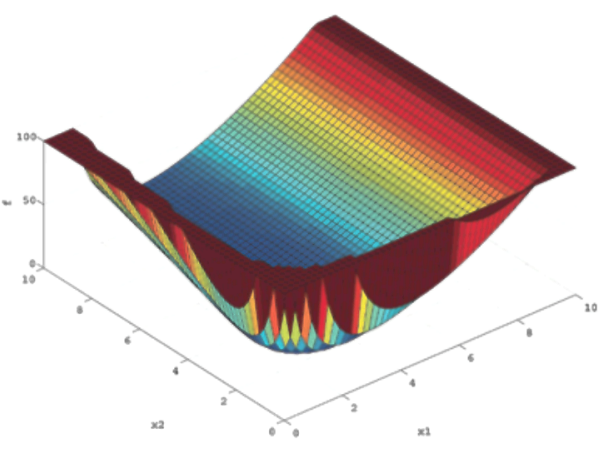

(a)

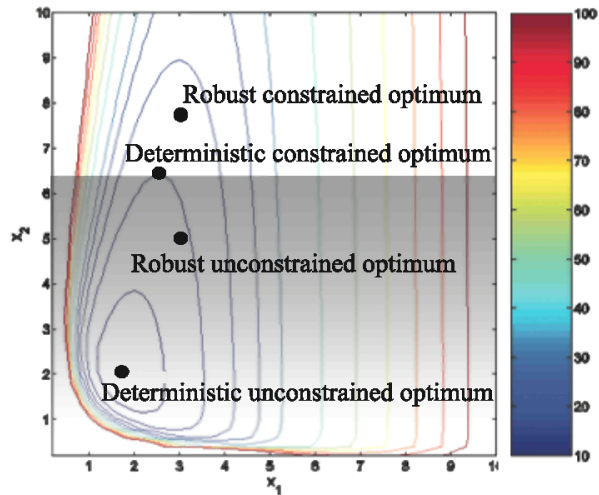

(b)

FIGURE 2. (a) Analytical test function; (b) Contour plot including optima

Figures 1(b) and (c) compare the differences in the optimisation algorithms and optimum evaluation for the deterministic and robust optimisation strategies. The difference in optimisation is the determination of the separate metamodels for $\mu_{y}$ and $\sigma_{y}$. This is done by Equation 2 when RSM is used as metamodelling technique; when Kriging is employed instead of RSM, an analytical derivation of $\mu_{y}$ and $\sigma_{y}$ is not possible. In this case we run a Monte Carlo Analysis (MCA) on the fitted metamodel as shown in Figure 1(c). The difference in the evaluation of the optimum $\mathbf{X}^{*}$ is that, in the deterministic case, this can be done by running one final FEM calculation. In case the robustness and reliability need to be assessed after optimisation, it is necessary to run an MCA using FEM calculations, which is quite time consuming.

\section{APPLICATION TO AN ANALYTICAL TEST FUNCTION}

The robust optimisation strategy will now be applied to the analytical test function presented in Figure 2(a). Figure 2(b) presents the contour of this objective function as well as a constraint. The constrained deterministic optimisation problem is:

$$
\min f=12+x_{1}^{2}+\frac{1+x_{2}^{2}}{x_{1}^{2}}+\frac{x_{1}^{2} x_{2}^{2}+100}{\left(x_{1} x_{2}\right)^{4}} ; \text { s.t. } g=6.5-x_{2} \leq 0 ; 0.1 \leq x_{1}, x_{2} \leq 10
$$

For the unconstrained deterministic optimisation model, the constraint $g$ is simply omitted. Both the unconstrained and constrained deterministic optima are presented in Figure 2(b).

The robust optimisation problem is modelled as follows:

$$
\min \mu_{f} ; \text { s.t. } \mu_{f}+3 \sigma_{f} \leq 50 ; \mu_{g}+3 \sigma_{g} \leq 0 ; 1 \leq x_{1}, x_{2} \sim N(\mu, 0.4) \leq 10
$$




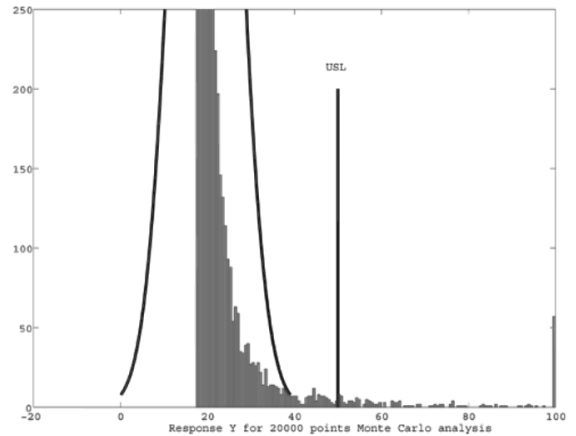

(a)

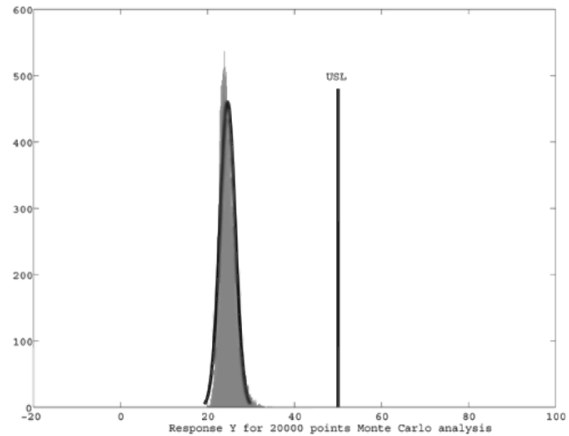

(b)

FIGURE 3. Response distributions: (a) Deterministic unconstrained optimum; (b) Robust unconstrained optimum

Again the unconstrained ( $g$ omitted) and the constrained problem have been optimised, this time using the robust optimisation strategy. 100 function evaluations are run for each optimisation. Both corresponding optima are again displayed in Figure 2(b).

After optimisation, the reliability of all optima has been evaluated using an MCA of 20000 function evaluations. Figure 3 compares the results of deterministic and robust unconstrained optimisation. The scrap rate has been reduced from $0.92 \%$ for the deterministic optimum to $<<0.005 \%$ for the robust optimum. The improvement of the robust optimisation strategy w.r.t. the deterministic one is even much more dramatic in constrained cases as depicted in Figure 4. For the deterministic optimum, the scrap rate due to violation of the constraint $g$ is $50.3 \%$ (Figure 4(b)). For the robust optimum, Figure $4(\mathrm{~d})$ shows that the scrap rate has been reduced to $0.1 \%$, which nicely corresponds to the $3 \sigma$ reliability level modelled in Equation 4.

\section{CONCLUSIONS}

Robustness, reliability, optimisation and Finite Element simulations are of major importance to improve product quality and reduce costs in the metal forming industry. In this paper, we proposed a robust optimisation strategy for metal forming processes. In addition to deterministic control variables, the strategy explicitly takes into account noise variables such as material variation and optimises probability distributions of objective function and constraints in order to achieve a robust and reliable metal forming process. The importance of including robustness during optimisation has been demonstrated by applying the robust optimisation strategy to an analytical test function: for constrained cases, deterministic optimisation will yield a scrap rate of about $50 \%$ whereas the robust optimisation strategy reduced this scrap rate to the demanded $3 \sigma$ reliability level. 


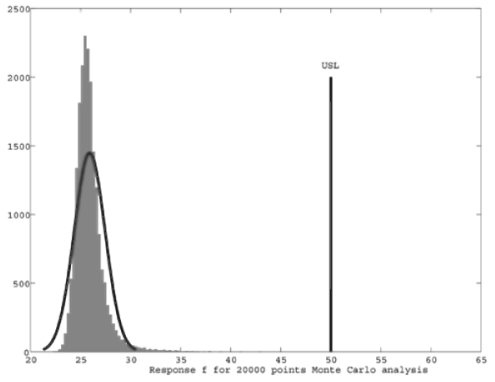

(a)

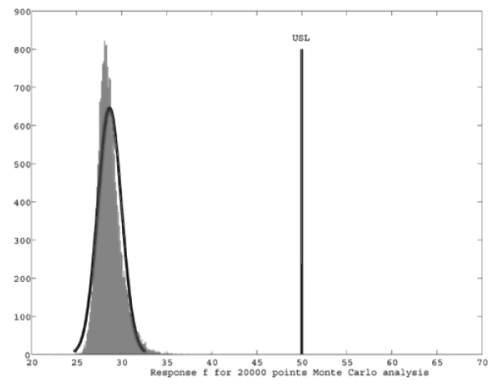

(c)

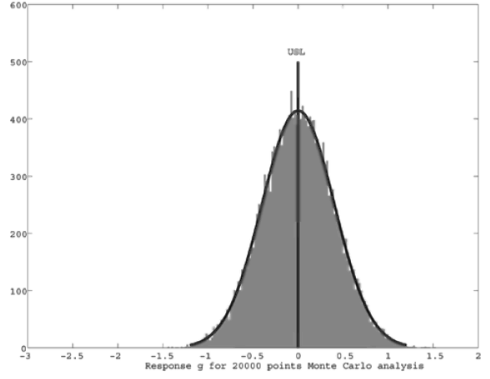

(b)

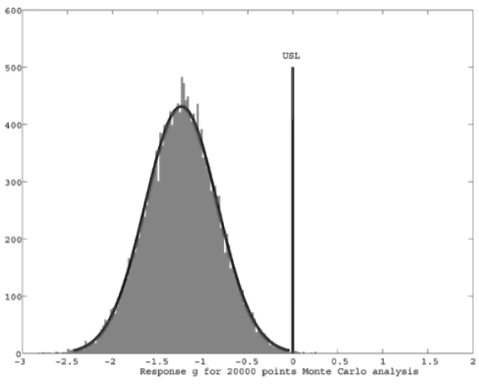

(d)

FIGURE 4. (a) Objective function distribution deterministic constrained optimum; (b) Constraint distribution deterministic constrained optimum; (c) Objective function distribution robust constrained optimum; (d) Constraint distribution robust constrained optimum

\section{ACKNOWLEDGMENTS}

This research has been carried out in the framework of the project "Optimisation of Forming Processes MC1.03162". This project is part of the research programme of the Netherlands Institute for Metals Research (NIMR). The industrial partners co-operating in this project are gratefully acknowledged for their useful contributions to this research.

\section{REFERENCES}

1. M. Bonte, A. van den Boogaard, and B. Carleer, "Optimising towards robust metal forming processes," in Proceedings of ESAFORM, Glasgow, UK, 2006, pp. 47-50.

2. R. Myers, and D. Montgomery, Response Surface Methodology: Process and Product Optimization Using Designed Experiments, John Wiley and Sons, Inc., New York, USA, 2002, $2^{\text {nd }}$ edn., ISBN 0-471-41255-4.

3. R. Myers, A. Khuri, and G. Vining, The American Statistician 46, 131-139 (1992).

4. V. Nair, Technometrics 34, 127-161 (1992).

5. M. Bonte, A. van den Boogaard, and J. Huétink, Submitted to: Structural and Multidisciplinary Optimization (2006). 